\title{
Increased Oxidative Stress and Imbalance in Antioxidant Enzymes in the Brains of Alloxan-Induced Diabetic Rats
}

\author{
Luciane B. Ceretta, ${ }^{1}$ Gislaine Z. Réus, ${ }^{1}$ Helena M. Abelaira, ${ }^{1}$ \\ Karine F. Ribeiro, ${ }^{1}$ Giovanni Zappellini, ${ }^{1}$ Francine F. Felisbino, ${ }^{2}$ \\ Amanda V. Steckert, ${ }^{1,2}$ Felipe Dal-Pizzol, ${ }^{2}$ and João Quevedo ${ }^{1}$
${ }^{1}$ Laboratório de Neurociências and Instituto Nacional de Ciência e Tecnologia Translacional em Medicina (INCT-TM), Programa de Pós-Graduação em Ciências da Saúde, Unidade Acadêmica de Ciências da Saúde, Universidade do Extremo Sul Catarinense, 88806-000 Criciúma, SC, Brazil
${ }^{2}$ Laboratório de Fisiopatologia Experimental and Instituto Nacional de Ciência e Tecnologia Translacional em Medicina (INCT-TM), Programa de Pós-Graduação em Ciências da Saúde, Unidade Acadêmica de Ciências da Saúde, Universidade do Extremo Sul Catarinense, 88806-000 Criciúma, SC, Brazil

Correspondence should be addressed to Gislaine Z. Réus, gislainezilli@hotmail.com

Received 14 December 2011; Revised 16 February 2012; Accepted 21 February 2012

Academic Editor: Dan Nemet

Copyright (C) 2012 Luciane B. Ceretta et al. This is an open access article distributed under the Creative Commons Attribution License, which permits unrestricted use, distribution, and reproduction in any medium, provided the original work is properly cited.

\begin{abstract}
Diabetes Mellitus (DM) is associated with pathological changes in the central nervous system (SNC) as well as alterations in oxidative stress. Thus, the main objective of this study was to evaluate the effects of the animal model of diabetes induced by alloxan on memory and oxidative stress. Diabetes was induced in Wistar rats by using a single injection of alloxan (150 mg/kg), and fifteen days after induction, the rats memory was evaluated through the use of the object recognition task. The oxidative stress parameters and the activity of antioxidant enzymes, superoxide dismutase (SOD), and catalase (CAT) were measured in the rat brain. The results showed that diabetic rats did not have alterations in their recognition memory. However, the results did show that diabetic rats had increases in the levels of superoxide in the prefrontal cortex, and in thiobarbituric acid reactive species (TBARS) production in the prefrontal cortex and in the amygdala in submitochondrial particles. Also, there was an increase in protein oxidation in the hippocampus and striatum, and in TBARS oxidation in the striatum and amygdala. The SOD activity was decreased in diabetic rats in the striatum and amygdala. However, the CAT activity was increased in the hippocampus taken from diabetic rats. In conclusion, our findings illustrate that the animal model of diabetes induced by alloxan did not cause alterations in the animals' recognition memory, but it produced oxidants and an imbalance between SOD and CAT activities, which could contribute to the pathophysiology of diabetes.
\end{abstract}

\section{Introduction}

Diabetes Mellitus (DM) is a heterogeneous metabolic disorder characterized by hyperglycemia [1]. In type 1 diabetes (DM1), which generally develops at a young age (children and early adulthood), the principal defect is an autoimmune-mediated destruction of pancreatic cells, leading to insulin deficiency [2]. In type 2 diabetes (DM2) the principal defect is insulin resistance, leading to a relative insulin deficiency in the liver and peripheral tissues, which leads to overt hyperglycaemia [3]. The hyperglycaemia in turn causes upto a fourfold increase in neuronal glucose, with intracellular glucose metabolism then leads to neuronal damage [4]. In addition to this, the current therapeutic strategies for DM2 are limited [5].

In both the human and animal models, DM is associated with pathological changes in the central nervous system (SNC) that lead to cognitive and affective deficits, and to an increased risk of brain vascular complications [3]. In the animal models of diabetes, several brain alterations have been described, such as increased hippocampal astrocytic reactivity, impaired synaptic plasticity, vascular changes, decreased 
dendritic complexity, and disturbed neurotransmission [6]. Recently, a significant body of evidence has accumulated to indicate that diabetes has detrimental effects on brain function. A number of investigations have been performed to indicate that memory loss is a consequence of both type I and type II diabetes [7]. Some authors have also reported a reduction in the length and a simplification of the dendritic trees of the hippocampal pyramidal cells in diabetic rodents [6]. There is evidence from the animal models showing that changes in dendritic morphology, probably associated with synaptic disturbances, correlate with alterations in memory and learning abilities [8]. Mitochondria are the principal source of reactive oxygen species (ROS) in cells, as the result of imperfectly coupled electron transport. Oxidative stress is widely accepted as playing a key mediatory role in the development and progression of diabetes and its complications, due to the increased production of free radicals and impaired antioxidant defenses [9]. Several mechanisms can contribute to increased oxidative stress in diabetic patients, especially chronic exposure to hyperglycemia. Accumulated evidence points out that hyperglycemia can lead to elevated ROS and reactive nitrogen species (RNS) production by the mitochondrial respiratory system [10], glucose autoxidation [11], activation of the polyol pathway [12], formation of advanced glycation end products (AGEs) [13], antioxidant enzyme inactivation [14] and an imbalance of glutathione redox status [15]. Hyperglycemia can promote an important oxidative imbalance, favoring the production of free radicals and the reduction of antioxidant defenses. At high concentrations, ROS/RNS can lead to damage to the major components of the cellular structure, including nucleic acids, proteins, amino acids, and lipids [16]. Such oxidative modifications in the diabetes condition would affect several cell functions, metabolism, and gene expression, which in turn can cause other pathological conditions [17].

It is important to note that the animal models of diabetes are very useful tools to gain new insights into human diabetes. Animal models induced by chemicals, such as alloxan, exhibit a syndrome of insulin resistance and type 2 diabetes [5]. Thus, the main objective of our study was to evaluate the effects of the animal model of diabetes induced by alloxan on the object recognition task and on the parameters of oxidative stress in the hippocampus, striatum, prefrontal cortex, and amygdala.

\section{Material and Methods}

2.1. Animals. Male Adult Wistar rats (60 days old) were obtained from the UNESC (Universidade do Extremo Sul Catarinense, Criciúma, SC, Brazil) breeding colony. They were housed five per cage with food and water available ad libitum and were maintained on a $12 \mathrm{~h}$ light/dark cycle (lights on at 7:00 a.m.). All experimental procedures involving animals were performed in accordance with the NIH Guide for the Care and Use of Laboratory Animals and the Brazilian Society for Neuroscience and Behavior (SBNeC) recommendations for animal care and with approval by the local Ethics Committee under protocol number 16/2010.
2.2. Diabetes Induction. Diabetes was induced in rats by using a single intraperitoneal injection of alloxan from Sigma Chemical Co. (St Louis, MO, USA) dissolved in a physiological saline $(0.9 \% \mathrm{NaCl})$ solution $(150 \mathrm{mg} / \mathrm{kg})$, whereas the control group received only a saline injection [18]. Both groups were injected after an $18 \mathrm{~h}$ fasting period $(60-70 \mathrm{mg} / \mathrm{dL}$ blood glucose). Fasting animals are more susceptible to alloxan probably due to partial protection by increased blood glucose [19]. All induced rats showed hyperglycemia (400-600 mg/dL) $48 \mathrm{~h}$ after alloxan administration. During the course of the experiment, the blood glucose level was monitored on a daily basis with commercial kits by performing a small puncture in the animals' tail. This methodology is quick and noninvasive, subjecting the rats to a negligible level of stress. At the end of the study, rats with glycemia between 400 and $600 \mathrm{mg} / \mathrm{dL}$ were considered diabetic [20]. Fifteen days after the induction of diabetes, based in previous studies [21], all rats were submitted to the object recognition task, after which the animals were killed by decapitation and a biochemical analysis was undertaken of the brain tissues.

2.3. Object Recognition Task. The object recognition task took place in a $40 \times 60 \mathrm{~cm}$ open field surrounded by $50 \mathrm{~cm}$ high walls made of ply wood with a frontal glass wall. The floor of the open field was divided into 12 equal rectangles by black lines. All animals (alloxan or saline; $n=10$ 15 animals per group) were submitted to a habituation session where they were allowed to freely explore the open field for $5 \mathrm{~min}$. No objects were placed in the box during the habituation trial. Twenty-four hours after habituation, training was conducted by placing the individual rats in the open field for $5 \mathrm{~min}$, in which two identical objects (objects A1 and A2; both being cubes) were positioned in two adjacent corners, $10 \mathrm{~cm}$ from the walls. In a short-term recognition memory test given $1.5 \mathrm{~h}$ after training, the rats explored the open field for $5 \mathrm{~min}$ in the presence of one familiar (A) and one novel (B, a rectangle) object. In a long-term recognition memory test given $24 \mathrm{~h}$ after training, the rats explored the open field for $5 \mathrm{~min}$ in the presence of one familiar (A) and one novel $(\mathrm{C}$, a pyramid with a square-shaped base) object. All objects had similar textures (smooth), colors (blue), and sizes (weight 150-200 g) but distinctive shapes. A recognition index calculated for each animal was calculated during the test session. It reports the ratio $\mathrm{TB} /(\mathrm{TA}+\mathrm{TB})(\mathrm{TA}=$ time spent exploring the familiar object $\mathrm{A} ; \mathrm{TB}=$ time spent exploring the novel object $\mathrm{B})$ and it reports the ratio $\mathrm{TC} /(\mathrm{TA}+\mathrm{TC})(\mathrm{TA}=$ time spent exploring the familiar object $\mathrm{A} ; \mathrm{TC}=$ time spent exploring the novel object C). Between trials, the objects were washed with $10 \%$ ethanol solution. Exploration was defined as sniffing (exploring the object $3-5 \mathrm{~cm}$ away from it) or touching the object with the nose and/or forepaws [21].

2.4. Oxidative Stress Parameters. Immediately after the object recognition task, the animals were sacrificed by decapitation and the following brain areas; the prefrontal cortex, amygdala, hippocampus and striatum $(n=4-6$ animals per 
group) were dissected according to the stereotaxic atlas [22] in ice-cold buffer, in a Petri dish. Submitochondrial particles were prepared in parallel from the four brain regions of each animal. For biochemical analysis in total tissue, the brain structures were rapidly frozen and stored at $-70^{\circ} \mathrm{C}$.

2.4.1. Mitochondrial Isolation. Rat brain homogenates were centrifuged at $700 \mathrm{~g}$ for $10 \mathrm{~min}$ to discard nuclei and cell debris and the pellet was then washed to enrich the supernatant that was centrifuged at $700 \mathrm{~g}$ for $10 \mathrm{~min}$. The obtained pellet, washed and resuspended in the same buffer, was considered to consist mainly of intact mitochondria able to carry out oxidative phosphorylation. The operations were carried out at $0-2{ }^{\circ} \mathrm{C}$. Submitochondrial particles (SMPs) were obtained by freezing and thawing (three cycles) of isolated mitochondria. For superoxide production measurements, SMP were washed twice with $140 \mathrm{mM} \mathrm{KCl,} 20 \mathrm{mM}$ Tris- $\mathrm{HCl}$ (pH 7.4) and suspended in the same medium [23].

2.4.2. Superoxide Production in Submitochondrial Particles of the Rat Brain. Superoxide production was determined in washed brain SMP using a spectrophotometric assay based on superoxide-dependent oxidation of epinephrine to adrenochrome at $37^{\circ} \mathrm{C}\left(€_{480 \mathrm{~nm}}=4.0 \mathrm{mM}^{-1} \mathrm{~cm}^{-1}\right)$. The reaction medium consisted of $0.23 \mathrm{M}$ mannitol, $0.07 \mathrm{M}$ sucrose, $20 \mathrm{mM}$ Tris-HCl ( $\mathrm{pH} 7.4$ ), SMP (0.3-1.0 mg protein $/ \mathrm{mL}), 0.1 \mu \mathrm{M}$ catalase, and $1 \mathrm{mM}$ epinephrine. NADH $(50 \mu \mathrm{M})$ and succinate $(7 \mathrm{mM})$ were used as substrates and rotenone $(1 \mu \mathrm{M})$ and antimycin $(1 \mu \mathrm{M})$ were added as specific inhibitors, respectively, to assay $\mathrm{O}_{2}{ }^{-}$production at the $\mathrm{NADH}$ dehydrogenase and at the ubiquinone-cytochrome $\mathrm{b}$ region. Superoxide dismutase (SOD) was used at $0.1-0.3 \mu \mathrm{M}$ final concentration to give assay specificity [24].

2.4.3. Thiobarbituric Acid Reactive Species Formation. To determine oxidative damage in lipid, we measured the formation of thiobarbituric acid reactive species (TBARS) during an acid-heating reaction, as previously described [25]. The samples were mixed with $1 \mathrm{~mL}$ of trichloroacetic acid $10 \%$ and $1 \mathrm{~mL}$ of thiobarbituric acid $0.67 \%$, and then heated in a boiling water bath for $30 \mathrm{~min}$. Malondialdehyde equivalents were determined in tissue and in submitochondrial particles of the rat brain spectrophotometrically by the absorbance at $532 \mathrm{~nm}$.

2.4.4. Carbonyl Protein Formation. Oxidative damage to proteins was assessed by the determination of carbonyl groups content based on the reaction with dinitrophenylhydrazine (DNPH), as previously described [26]. Proteins were precipitated by the addition of $20 \%$ trichloroacetic acid and were redissolved in DNPH. The absorbance was monitored spectrophotometrically at $370 \mathrm{~nm}$.

2.4.5. Superoxide Dismutase Activity. This method for the assay of superoxide dismutase (SOD) activity is based on the capacity of pyrogallol to autoxidize, a process highly dependent on $\mathrm{O}_{2}^{-2}$; a substrate for SOD [27]. The inhibition of autoxidation of this compound thus occurs when
SOD is present, and the enzymatic activity can be then indirectly assayed spectrophotometrically at $420 \mathrm{~nm}$, using a double-beam spectrophotometer with temperature control. A calibration curve was performed using purified SOD as the standard, in order to calculate the specific activity of SOD present in the samples. A 50\% inhibition of pyrogallol autoxidation is defined as 1 unit of SOD, and the specific activity is represented as units per $\mathrm{mg}$ of protein.

2.4.6. Catalase Activity. The catalase (CAT) activity was assayed using a double-beam spectrophotometer with temperature control. This method is based on the disappearance of $\mathrm{H}_{2} \mathrm{O}_{2}$ at $240 \mathrm{~nm}$ in a reaction medium containing $20 \mathrm{mM}$ $\mathrm{H}_{2} \mathrm{O}_{2}, 0.1 \%$ Triton $\mathrm{X}-100,10 \mathrm{mM}$ potassium phosphate buffer, $\mathrm{pH} 7.0$, and $0.1-0.3 \mathrm{mg}$ protein $/ \mathrm{mL}$ [28]. One CAT unit is defined as $1 \mathrm{~mol}$ of hydrogen peroxide consumed per minute, and the specific activity is reported as units per mg protein.

2.4.7. Protein Determination. All biochemical measures were normalized to the protein content with bovine albumin as standard [29].

2.5. Statistical Analysis. In the open field test, the differences between training test sessions were analyzed by the paired Student's $t$-test. Data for recognition indexes are reported as median \pm interquartile ranges (25 and 75). Comparisons among groups were performed using the Kruskal-Wallis test followed by Mann-Whitney test when necessary. The oxidative stress parameters were analyzed by Student's $t$-test for unpaired data and are reported as mean \pm S.E.M. $P$ values less than 0.05 were considered to be statistically significant.

\section{Results}

As depicted in Figure 1, in the object recognition task, no statistical differences were observed in the saline or alloxan groups in the training session $(P>0.05)$. In the control rats group, $1.5 \mathrm{~h}$ after the training session (short-term recognition memory), we observed an increase in the recognition index compared to the training session, and $24 \mathrm{~h}$ after the training session (long-term recognition memory) there was an increase in the recognition index, compared to the training session or to the short-term memory tests $(P<0.05$; Figure 1). In the diabetic rats induced by alloxan, there was an increase in the recognition index after $24 \mathrm{~h}$, but not $1.5 \mathrm{~h}$ after the training session $(P<0.05$; Figure 1$)$.

In diabetic rats, there was an increase in the superoxide submitochondrial particles in the prefrontal cortex $(P<$ 0.05 ; Figure $2(\mathrm{a})$ ) and an increase in the TBARS submitochondrial particles in the prefrontal cortex and amygdala (Figure 2(b)). In diabetic rats it was shown that there was an increase in the carbonyl proteins in the hippocampus and striatum $(P<0.05$; Figure $3(\mathrm{a}))$ and an increase in the TBARS oxidation in the striatum and amygdala $(P<0.05$; Figure 3(b)). The SOD activity was decreased in diabetic rats in the striatum and amygdala $(P<0.05$; Figure $4(\mathrm{a}))$. 


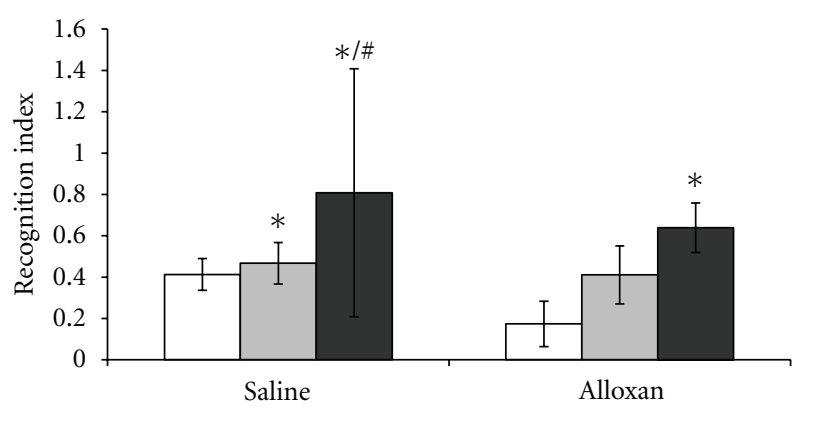

$$
\begin{array}{ll}
\square & \text { Training } \\
\square & 1.5 \mathrm{~h} \text { retention } \\
\square & 24 \mathrm{~h} \text { retention }
\end{array}
$$

FIGURE 1: The effects of the animal model of diabetes induced by alloxan on the object recognition task. Results are reported as median \pm interquartile ranges of the recognition indexes in training and short- and long-term memory retention test trials. $N=10-15$ per group, ${ }^{*} P<0.05$ difference from the training session and ${ }^{\#} P<$ 0.05 difference from the training from $1.5 \mathrm{~h}$ retention, according to Kruskal-Wallis test.

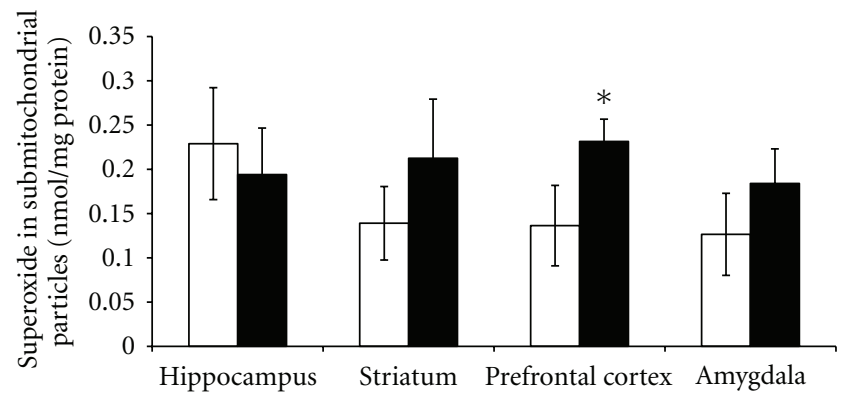

(a)

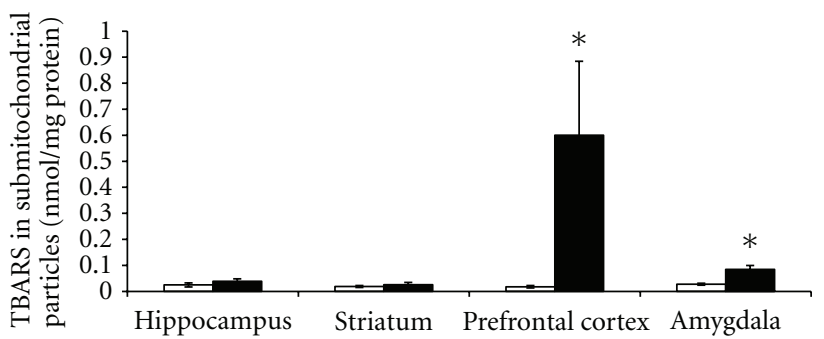

- Control

- Alloxan

(b)

Figure 2: The effects of the animal model of diabetes induced by alloxan on the superoxide (a) and TBARS (b) production in submitochondrial particles in the hippocampus, striatum, prefrontal cortex and amygdala. Results are reported as mean \pm S.E.M. $N=4$ 6 per group, ${ }^{*} P<0.05$ difference from the saline group, according to the Student $t$-test.

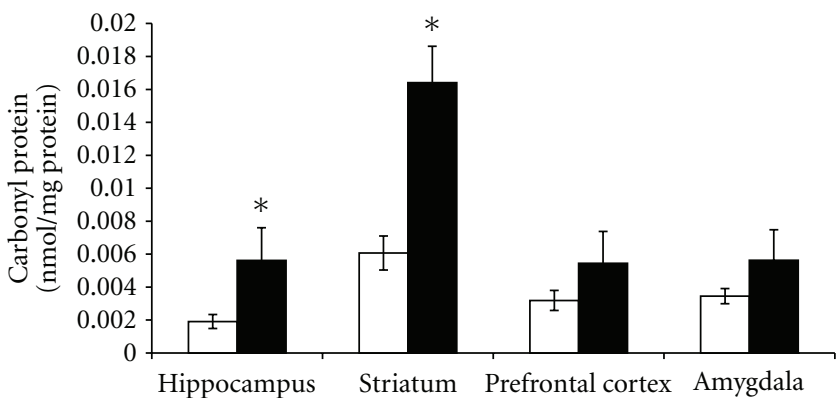

(a)

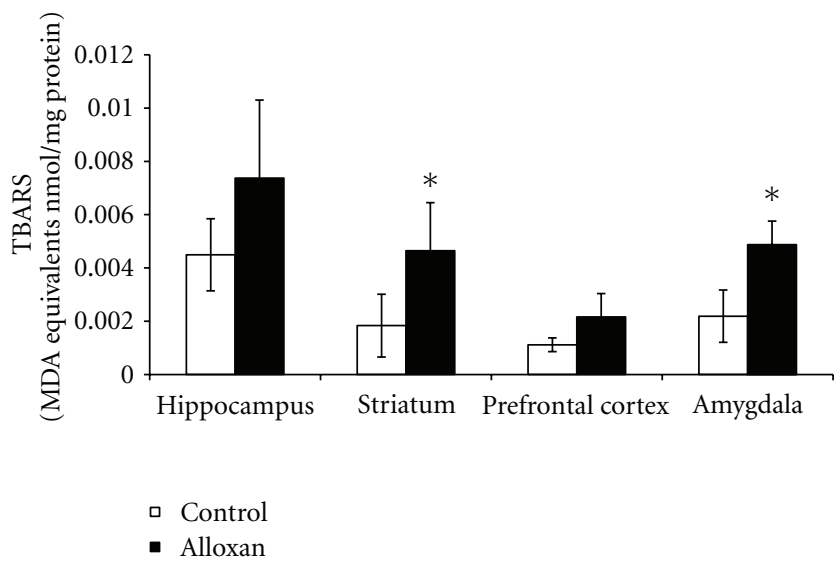

(b)

Figure 3: The effects of the animal model of diabetes induced by alloxan on TBARS production (a) and carbonyl formation (b) in the hippocampus, striatum, prefrontal cortex, and amygdala. Results are reported as mean \pm S.E.M. $N=4-6$ per group, ${ }^{*} P<0.05$ difference from the saline group, according to the Student's $t$-test.

However, the CAT activity was increased in the hippocampus from diabetic rats $(P<0.05$; Figure $4(\mathrm{~b}))$.

\section{Discussion}

Experimental diabetes models can be induced by chemicals that selectively destroy the insulin-producing $\beta$-cells in the pancreas [30]. One of the most commonly used chemicals is alloxan. This drug induces diabetes by intracellular generation of ROS formed in a cyclic reaction involving alloxan and its reduced product called dialuric acid [18], with subsequent inhibition of insulin synthesis and secretion.

Recently, a significant body of evidence has accumulated to indicate that diabetes has detrimental effects on brain function. In fact, hypoglycemia and diabetes insults are related with damage in the hippocampus and hypothalamus, which are the brain areas associated with memory and plasticity functions [31]. A number of investigations have been performed to indicate that memory loss is a consequence of both type I and type II diabetes [3, 32]. However, the exact mechanism(s) as to how diabetic conditions could affect memory activity remains to be fully characterized. In the present study our results showed that in diabetic rats, there was an increase in the recognition index $24 \mathrm{~h}$ after 


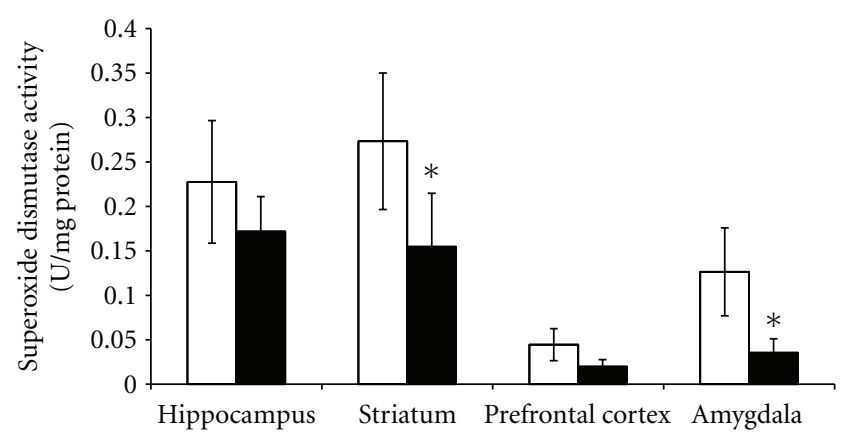

(a)

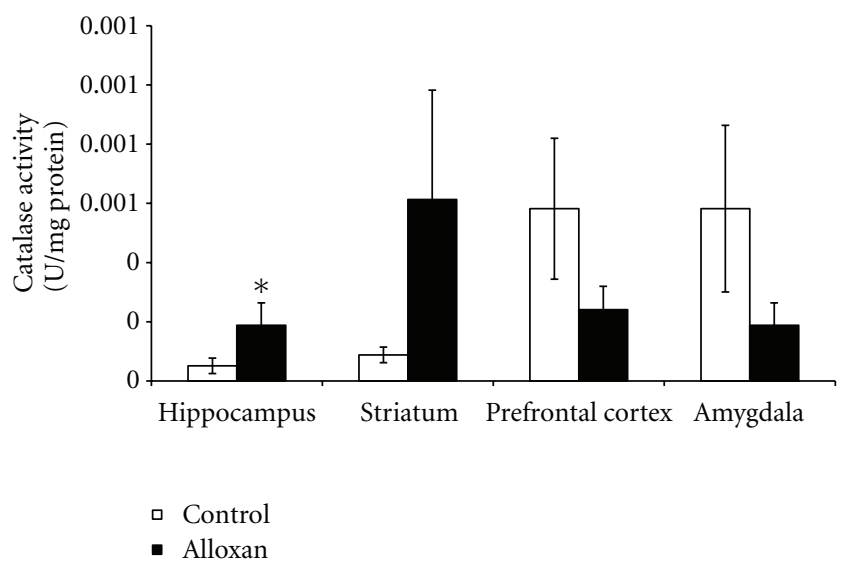

(b)

Figure 4: The effects of the animal model of diabetes induced by alloxan on the superoxide dismutase (a) and catalase (b) activities in the hippocampus, striatum, prefrontal cortex, and amygdala. Results are reported as mean \pm S.E.M. $N=4-6$ per group, ${ }^{*} P<$ 0.05 difference from the saline group, according to the Student's $t$ test.

the training session, indicating that diabetic rats did not alter recognition memory, when subjected to the object recognition task. Contrary to this, another study showed a significant reduction of memory formation, evaluated in the passive avoidance test in streptozotocin-induced diabetic mice [7]. The authors suggest that the overexpressed $\beta$ amyloid precursor might be one of the underlying factors causing memory deficit.

Differences between these studies could be related with the animal model of diabetes and tests used to evaluate memory. Although some studies have shown that oxidative stress induces significant deficits in cognitive performance (learning ability and memory retention) [33], in the present findings we did not show this correlation. It is important to note that during the formation of memories the activation of specific receptors and of several molecular cascades is required to convert extracellular signals that lead to changes in neuronal connectivity [34], which were probably not altered in the brains of alloxan-induced diabetic rats. For example, we recently demonstrated that brainderived neurotrophic factor (BDNF) levels did not alter in the hippocampus from alloxan-induced diabetic rats [35].
BDNF is a neurotrophin which has an important role in hippocampal-dependent forms of memory [36]. Thus, the findings of the present study on recognition memory could be related, at least in part, because the animal model of diabetes induced by alloxan did not alter BDNF levels.

A previous study showed that alloxan-induced diabetes is associated with changes in the uptake of insulin by the brain, which includes increased binding to the capillary bed comprising the blood brain barrier and increased transport across the blood brain barrier [37]. Moreover, studies related that oxidative stress impacts several brain areas, such as the forebrain, cerebellum, and brain stem $[31,38]$. Thus, in the present study we evaluated oxidative stress parameters in different brain areas, namely, the hippocampus, striatum, prefrontal cortex, and amygdala. Uncontrolled ROS production could lead to damage in cellular macromolecules (DNA, lipids, and protein) and other small antioxidant molecules [39], contributing to the progress of diabetic complications. Still, research indicates that obesity and hyperglycemia are associated with increases in the ROS production [40, 41]. In the present study we observed an increase in the superoxides in the prefrontal cortex and an increase in TBARS in the prefrontal cortex and amygdala in submitochondrial particles. Diabetes causes mitochondrial superoxide overproduction and this increased superoxide production is the major mediator of diabetes tissue damage [41]. In fact, in diabetic cells with a high intracellular glucose concentration, there is more glucose-derived pyruvate being oxidized in the tricarboxylic acid cycle, increasing the flux of electron donors (NADH and FADH2) into the electron transport chain [42]. Thus, electron transfer inside complex III is blocked [43], causing the electrons to use coenzyme Q as a backup, which donates the electrons one at a time to molecular oxygen, thereby generating superoxide [42]. The mitochondria are an organelle and have the ability to generate superoxides at complexes I and III [44, 45]. In addition to this, in brain tissue, complexes I and III have been attributed to major ROS production $[44,45]$. In fact, a study from our group demonstrated that in alloxan-induced diabetic rats there were alterations in the mitochondrial respiratory chain [20]. Thus we suggest that alloxan-induced diabetic-like symptoms may provide a useful animal model to test the hypothesis of the involvement of oxidative stress in diabetes. Our results also showed an increase in carbonyl protein in the hippocampus, and amygdala and an increase in TBARS oxidation in the striatum and amygdala from diabetic animals. We discovered from our data that the TBARS levels in mitochondrial particles and in tissues were different, but our analysis was conducted in different areas, involving separate samples from submitochondrial particles and another from tissue. It is known that the mitochondria is a major producer of ROS [42], which in turn causes damage in lipids, which may explain the increase in TBARS in submitochondrial particles in the prefrontal cortex. Recently, Chang et al. [46] showed an increase in the carbonyl protein in the renal tissues from diabetic animals induced by streptozotocin. Additionally, similar to our findings, the TBARS index was increased in the liver of rats that had received a single injection of alloxan $(150 \mathrm{mg} / \mathrm{kg})$ [30]. Also, 
there was shown to be an increase in the TBARS in the plasma and hippocampus from diabetic animals induced by streptozotocin [47]. Recently, [48] investigated the effects of agmatine, an antihyperglycemic and antioxidant, on MDA and glutathione levels in the cerebral cortex and hippocampus from diabetic rats. The authors found that treatment with agmatine reduced oxidative stress markers in the brain of diabetic rats. In addition, [48] showed that brain regions differ in their response to oxidative stress in obese $\mathrm{db} / \mathrm{db}$ mice. Still, silibinin, a compound with antioxidant properties, provided efficient neuroprotection in these diabetic rats [48]. Thus, this suggests that diabetes is related to oxidative stress in the brain.

The present findings showed that SOD activity decreased in the striatum and amygdala. On the other hand, the CAT activity increased in the hippocampus in diabetic animals. SOD is a protective enzyme that can selectively scavenge the superoxide anion radical $\left(\mathrm{O}_{2}^{-\cdot}\right)$ by catalyzing its dismutation to hydrogen peroxide $\left(\mathrm{H}_{2} \mathrm{O}_{2}\right)$ [49]. CAT catalyzes degradation of $\mathrm{H}_{2} \mathrm{O}_{2}$ to water and $\mathrm{O}_{2}$. Another study showed that SOD and CAT activities were increased in the livers from alloxan-induced diabetic rats [31]. Also, Amer et al. [50] demonstrated that polymorphisms of glutathione Stransferase (an antioxidant enzyme) genes GSTM1 and GSTT1 were associated with an increased risk of type 2 DM. Interestingly, Di Naso et al. [51] reported that exogenous antioxidant copper zinc superoxide dismutase ( $\mathrm{Cu} / \mathrm{Zn}$ SOD) decreased liver peroxidation and increased nitric oxide synthase (NOS) in diabetic rats. Moreover, Gibson et al. [52] showed that N-acetylcysteine (NAC), a biosynthetic precursor of the antioxidant glutathione, reduced thrombotic propensity in type 2 diabetes patients, suggesting that this effect occurred by increasing the platelet antioxidant status as a result of elevated glutathione synthesis.

An imbalance in the SOD/CAT ratio indicates the generation of reactive species [53], which were reported in the present study. In this context, the effects of alloxan on SOD/CAT turnover (by increasing CAT and decreasing SOD activity) may result in antioxidant effects. An imbalance in the SOD/CAT ratio indicates the generation of reactive species, which were reported in the present study. Thus, considering the pathophysiology of diabetes, and the results presented in the present work, it is sensible to suggest that differences in oxidative stress parameters may be related, at least in part, with brain metabolism. Another study from our group also showed a decrease in SOD and an increase in CAT activities in the brain of rats submitted to the chronic mild stress procedure [54]. In fact, studies have reported a relationship between diabetes and stress $[55,56]$ inclusively, alloxan-induced diabetic rats presented depressive-like behaviour [20].

The cause of the overall oxidative imbalance demonstrated in our study may be due to mitochondrial dysfunction. Recently, it was shown that alloxan-induced diabetic rats presented alterations in the mitochondrial respiratory chain, creatine kinase, and citrate synthase activities [20]. However, mitochondrial alteration could occur by oxidative imbalance. In fact, ROS causes damage in the mitochondrial oxidative phosphorilation [56]. In addition, Bhattacharya et al. [57] reported decreased mitochondrial membrane potential, enhanced cytochrome c release, reciprocal regulation of the Bcl-2 family, and increases of caspases 3 and 9 in alloxan-induced diabetes. The authors also showed that treatment with D-saccharic acid 1,4-lactone, a derivative of D-glucaric acid which has antioxidant properties, counteracted these changes [57].

\section{Conclusions}

To our knowledge our data describes for the first time, the effects of the animal model of diabetes induced by alloxan on memory and oxidative stress parameters in the rat brain. In conclusion, alloxan-induced diabetes did not alter recognition memory, but induced oxidative damage and an imbalance between antioxidant enzymes, contributing, at least in part, to the pathophysiology of diabetes.

\section{Acknowledgments}

This study was supported in part by grants from "Conselho Nacional de Desenvolvimento Científico e Tecnológico" (CNPq-Brazil-J. Quevedo and F. Dal-Pizzol), from the Instituto Cérebro e Mente (J. Quevedo) and UNESC (J. Quevedo and F. Dal-Pizzol). J. Quevedo and F. Dal-Pizzol are recipients of $\mathrm{CNPq}$ (Brazil) Productivity Fellowships. G. Z. Réus and A. V. Steckert are holders of a CAPES studentship. Finally, the are grateful to Luciano K. Jornada for assisting in the statistical analysis.

\section{References}

[1] G. I. Robles and D. Singh-Franco, "A review of exenatide as adjunctive therapy in patients with type 2 diabetes," Drug Design, Development and Therapy, no. 3, pp. 219-240, 2009.

[2] G. J. Biessels, L. P. Van der Heide, A. Kamal, R. L. A. W. Bleys, and W. H. Gispen, "Ageing and diabetes: implications for brain function," European Journal of Pharmacology, vol. 441, no. 1-2, pp. 1-14, 2002.

[3] G. J. Biessels and W. H. Gispen, "The impact of diabetes on cognition: what can be learned from rodent models?" Neurobiology of Aging, vol. 26, no. 1, supplement, pp. S36-S41, 2005.

[4] D. R. Tomlinson and N. J. Gardiner, "Glucose neurotoxicity," Nature Reviews Neuroscience, vol. 9, no. 1, pp. 36-45, 2008.

[5] K. Srinivasan and P. Ramarao, "Animal models in type 2 diabetes research: an overview," Indian Journal of Medical Research, vol. 125, no. 3, pp. 451-472, 2007.

[6] A. M. Magariños and B. S. McEwen, "Experimental diabetes in rats causes hippocampal dendritic and synaptic reorganization and increased glucocorticoid reactivity to stress," Proceedings of the National Academy of Sciences of the United States of America, vol. 97, no. 20, pp. 11056-11061, 2000.

[7] S. W. Jung, O. K. Han, and S. J. Kim, "Increased expression of $\beta$ amyloid precursor gene in the hippocampus of streptozotocin-induced diabetic mice with memory deficit and anxiety induction," Journal of Neural Transmission, vol. 117, no. 12, pp. 1411-1418, 2010.

[8] B. Kolb, J. Cioe, and W. Comeau, "Contrasting effects of motor and visual spatial learning tasks on dendritic arborization and 
spine density in rats," Neurobiology of Learning and Memory, vol. 90, no. 2, pp. 295-300, 2008.

[9] A. Ceriello, "New insights on oxidative stress and diabetic complications may lead to a "causal" antioxidant therapy," Diabetes Care, vol. 26, no. 5, pp. 1589-1596, 2003.

[10] T. Nishikawa and E. Araki, "Impact of mitochondrial ROS production in the pathogenesis of diabetes mellitus and its complications," Antioxidants and Redox Signaling, vol. 9, no. 3, pp. 343-353, 2007.

[11] M. A. Yorek, "The role of oxidative stress in diabetic vascular and neural disease," Free Radical Research, vol. 37, no. 5, pp. 471-480, 2003.

[12] N. E. Cameron, M. A. Cotter, and T. C. Hohman, "Interactions between essential fatty acid, prostanoid, polyol pathway and nitric oxide mechanisms in the neurovascular deficit of diabetic rats," Diabetologia, vol. 39, no. 2, pp. 172-182, 1996.

[13] V. M. Monnier, "Intervention against the Maillard reaction in vivo," Archives of Biochemistry and Biophysics, vol. 419, no. 1, pp. 1-15, 2003.

[14] A. C. Maritim, R. A. Sanders, and J. B. Watkins, "Diabetes, oxidative stress, and antioxidants: a review," Journal of Biochemical and Molecular Toxicology, vol. 17, no. 1, pp. 24-38, 2003.

[15] H. Kaneto, J. Fujii, K. Suzuki et al., "DNA cleavage induced by glycation of $\mathrm{Cu}, \mathrm{Zn}$-superoxide dismutase," Biochemical Journal, vol. 304, no. 1, pp. 219-225, 1994.

[16] M. Valko, D. Leibfritz, J. Moncol, M. T. D. Cronin, M. Mazur, and J. Telser, "Free radicals and antioxidants in normal physiological functions and human disease," The International Journal of Biochemistry and Cell Biology, vol. 39, no. 1, pp. 4484, 2007.

[17] I. S. Young and J. V. Woodside, "Antioxidants in health and disease," Journal of Clinical Pathology, vol. 54, no. 3, pp. 176186, 2001.

[18] G. A. Behr, E. G. Da Silva, A. R. Ferreira, C. T. S. Cerski, F. Dal-Pizzol, and J. C. F. Moreira, "Pancreas $\beta$-cells morphology, liver antioxidant enzymes and liver oxidative parameters in alloxan-resistant and alloxan-susceptible Wistar rats: a viable model system for the study of concepts into reactive oxygen species," Fundamental and Clinical Pharmacology, vol. 22, no. 6, pp. 657-666, 2008.

[19] T. Szkudelski, "The mechanism of alloxan and streptozotocin action in B cells of the rat pancreas," Physiological Research, vol. 50, no. 6, pp. 537-546, 2001.

[20] L. B. Ceretta, G. Z. Réus, G. T. Rezin, G. Scaini, E. L. Streck, and J. Quevedo, "Brain energy metabolism parameters in an animal model of diabetes," Metabolic Brain Disease, vol. 25, no. 4, pp. 391-396, 2010.

[21] T. Barichello, M. R. Martins, A. Reinke et al., "Behavioral deficits in sepsis-surviving rats induced by cecal ligation and perforation," Brazilian Journal of Medical and Biological Research, vol. 40, no. 6, pp. 831-837, 2007.

[22] G. Paxinos and C. Watson, The Rat Brain: Stereotaxic Coordinates, Academic Press, Sydney, Australia, 2nd edition, 1986.

[23] A. Boveris, N. Oshino, and B. Chance, "The cellular production of hydrogen peroxide," Biochemical Journal, vol. 128, no. 3, pp. 617-630, 1972.

[24] A. Boveris, "Determination of the production of superoxide radicals and hydrogen peroxide in mitochondria," Methods in Enzymology, vol. 105, pp. 429-435, 1984.

[25] H. H. Draper and M. Hadley, "Malondialdehyde determination as index of lipid peroxidation," Methods in Enzymology, vol. 186, pp. 421-431, 1990.
[26] R. L. Levine, J. A. Williams, E. R. Stadtman, and E. Shacter, "Carbonyl assays for determination of oxidatively modified proteins," Methods in Enzymology, vol. 233, pp. 346-357, 1994.

[27] J. V. Bannister and L. Calabrese, "Assays for superoxide dismutase," in Methods of Biochemical Analysis, vol. 32, pp. 279-312, John Wiley \& Sons, New York, NY, USA, 1987.

[28] H. Aebi, "Catalase in vitro," Methods in Enzymology, vol. 105, no. C, pp. 121-126, 1984.

[29] O. H. Lowry, N. J. Rosebrough, A. L. Farr, and R. J. Randall, "Protein measurement with the Folin phenol reagent," The Journal of Biological Chemistry, vol. 193, no. 1, pp. 265-275, 1951.

[30] T. Szkudelski, "The mechanism of alloxan and streptozotocin action in B cells of the rat pancreas," Physiological Research, vol. 50, no. 6, pp. 537-546, 2001.

[31] A. J. Bree, E. C. Puente, D. Daphna-Iken, and S. J. Fisher, "Diabetes increases brain damage caused by severe hypoglycemia," American Journal of Physiology-Endocrinology and Metabolism, vol. 297, no. 1, pp. E194-E201, 2009.

[32] J. Beauquis, F. Homo-Delarche, M. H. Giroix et al., "Hippocampal neurovascular and hypothalamic-pituitary-adrenal axis alterations in spontaneously type 2 diabetic GK rats," Experimental Neurology, vol. 222, no. 1, pp. 125-134, 2010.

[33] K. Fukui, N. O. Omoi, T. Hayasaka et al., "Cognitive impairment of rats caused by oxidative stress and aging, and its prevention by vitamin E," Annals of the New York Academy of Sciences, vol. 959, pp. 275-284, 2002.

[34] S. Laroche, "Cellular and molecular mechanisms of memory," Biologie Aujourd'hui, vol. 204, no. 2, pp. 93-102, 2010.

[35] L. B. Ceretta, G. Z. Réus, R. B. Stringari et al., "Imipramine treatment reverses depressive-like behavior in alloxan-diabetic rats," Diabetes/Metabolism Research and Reviews, vol. 28, no. 2, pp. 139-144, 2012.

[36] B. Lu and K. Martinowich, "Cell biology of BDNF and its relevance to schizophrenia," Novartis Foundation Symposium, vol. 289, pp. 119-129, 2008.

[37] W. A. Banks, J. B. Jaspan, and A. J. Kastin, "Effect of diabetes mellitus on the permeability of the blood-brain barrier to insulin," Peptides, vol. 18, no. 10, pp. 1577-1584, 1997.

[38] M. L. Haces, T. Montiel, and L. Massieu, "Selective vulnerability of brain regions to oxidative stress in a non-coma model of insulin-induced hypoglycemia," Neuroscience, vol. 165, no. 1, pp. 28-38, 2010.

[39] B. Halliwell, "Antioxidant defence mechanisms: from the beginning to the end (of the beginning)," Free Radical Research, vol. 31, no. 4, pp. 261-272, 1999.

[40] M. Brownlee, "Biochemistry and molecular cell biology of diabetic complications," Nature, vol. 414, no. 6865, pp. 813$820,2001$.

[41] S. Furukawa, T. Fujita, M. Shimabukuro et al., "Increased oxidative stress in obesity and its impact on metabolic syndrome," Journal of Clinical Investigation, vol. 114, no. 12, pp. 1752-1761, 2004.

[42] F. Giacco and M. Brownlee, "Oxidative stress and diabetic complications," Circulation Research, vol. 107, no. 9, pp. 10581070, 2010.

[43] B. L. Trumpower, "The protonmotive Q cycle. Energy transduction by coupling of proton translocation to electron transfer by the cytochrome bc1 complex," Journal of Biological Chemistry, vol. 265, no. 20, pp. 11409-11412, 1990.

[44] A. Boveris, E. Cadenas, and A. O. M. Stoppani, "Role of ubiquinone in the mitochondrial generation of hydrogen peroxide," Biochemical Journal, vol. 156, no. 2, pp. 435-444, 1976. 
[45] J. F. Turrens and A. Boveris, "Generation of superoxide anion by the NADH dehydrogenase of bovine heart mitochondria," Biochemical Journal, vol. 191, no. 2, pp. 421-427, 1980.

[46] C.-C. Chang, C.-Y. Chang, Y.-T. Wu, J.-P. Huang, T.-H. Yen, and L.-M. Hung, "Resveratrol retards progression of diabetic nephropathy through modulations of oxidative stress, proinflammatory cytokines, and AMP-activated protein kinase," Journal of Biomedical Science, vol. 18, no. 1, article 47, 2011.

[47] C. A. Y. Wayhs, V. Manfredini, A. Sitta et al., "Protein and lipid oxidative damage in streptozotocin-induced diabetic rats submitted to forced swimming test: the insulin and clonazepam effect," Metabolic Brain Disease, vol. 25, no. 3, pp. 297-304, 2010.

[48] G. Marrazzo, P. Bosco, F. La Delia et al., "Neuroprotective effect of silibinin in diabetic mice," Neuroscience Letters, vol. 504, no. 3, pp. 252-256, 2011.

[49] I. Fridovich, "Superoxide dismutases: regularities and irregularities," Harvey lectures, vol. 79, pp. 51-75, 1983.

[50] M. A. Amer, M. H. Ghattas, D. M. Abo-Elmatty, and S. H. Abou-El-Ela, "Influence of glutathione S-transferase polymorphisms on type-2 diabetes mellitus risk," Genetics and Molecular Research, vol. 10, no. 4, pp. 3788-3730, 2011.

[51] F. C. Di Naso, A. Simoes Dias, M. Porawski, and N. A. P. Marroni, "Exogenous superoxide dismutase: action on liver oxidative stress in animals with streptozotocin-induced diabetes," Experimental Diabetes Research, vol. 2011, Article ID 754132, 6 pages, 2011.

[52] K. R. Gibson, T. J. Winterburn, F. Barrett, S. Sharma, S. M. MacRury, and I. L. Megson, "Therapeutic potential of Nacetylcysteine as an antiplatelet agent in patients with type-2 diabetes," Cardiovascular Diabetology, vol. 10, article 43, 2011.

[53] T. M. Michel, J. Thome, D. Martin et al., "Cu, Zn- And Mn-superoxide dismutase levels in brains of patients with schizophrenic psychosis," Journal of Neural Transmission, vol. 111, no. 9, pp. 1191-1201, 2004.

[54] G. Lucca, C. M. Comim, S. S. Valvassori et al., "Effects of chronic mild stress on the oxidative parameters in the rat brain," Neurochemistry International, vol. 54, no. 5-6, pp. 358362, 2009.

[55] S. H. Golden, M. Lazo, M. Carnethon et al., "Examining a bidirectional association between depressive symptoms and diabetes," Journal of the American Medical Association, vol. 299, no. 23, pp. 2751-2759, 2008.

[56] C. Shen, P. Findley, R. Banerjea, and U. Sambamoorthi, "Depressive disorders among cohorts of women veterans with diabetes, heart disease, and hypertension," Journal of Women's Health, vol. 19, no. 8, pp. 1475-1486, 2010.

[57] S. Bhattacharya, P. Manna, R. Gachhui, and P. C. Sil, "Dsaccharic acid-1,4-lactone ameliorates alloxan-induced diabetes mellitus and oxidative stress in rats through inhibiting pancreatic beta-cells from apoptosis via mitochondrial dependent pathway," Toxicology and Applied Pharmacology, vol. 257, no. 2, pp. 272-283, 2011. 


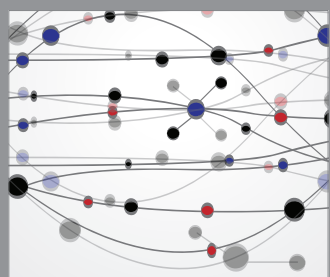

The Scientific World Journal
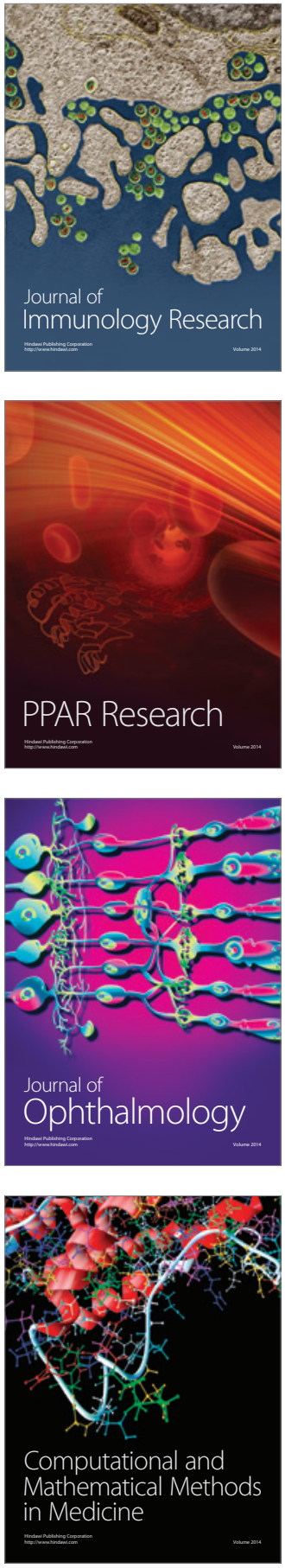

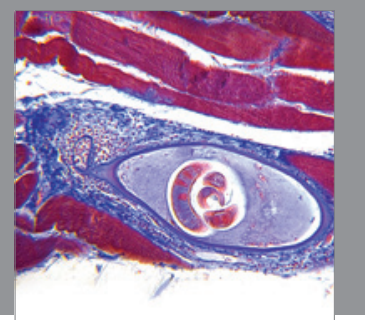

Gastroenterology

Research and Practice
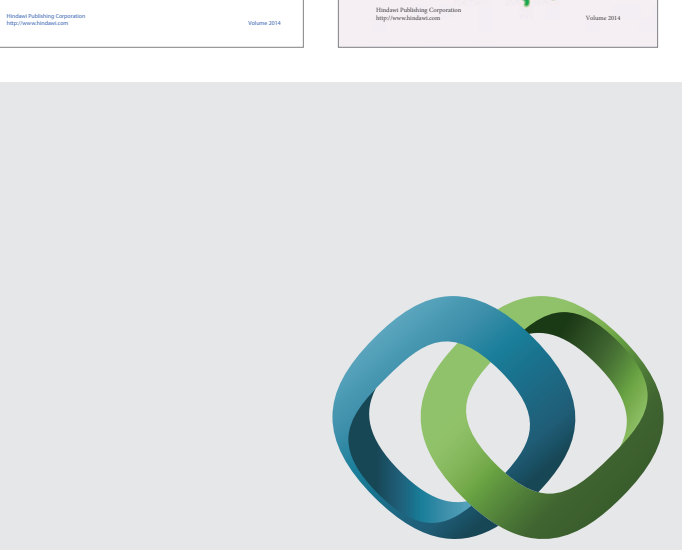

\section{Hindawi}

Submit your manuscripts at

http://www.hindawi.com
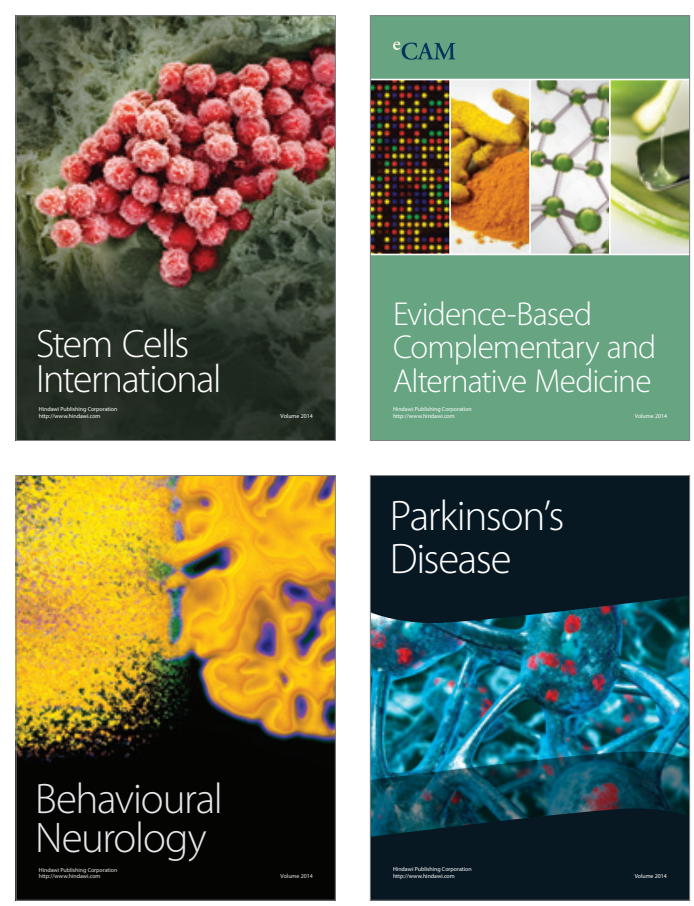

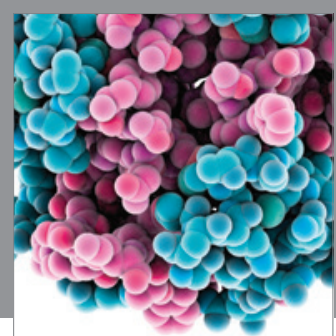

Journal of
Diabetes Research

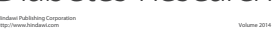

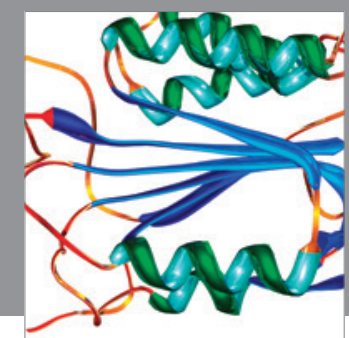

Disease Markers
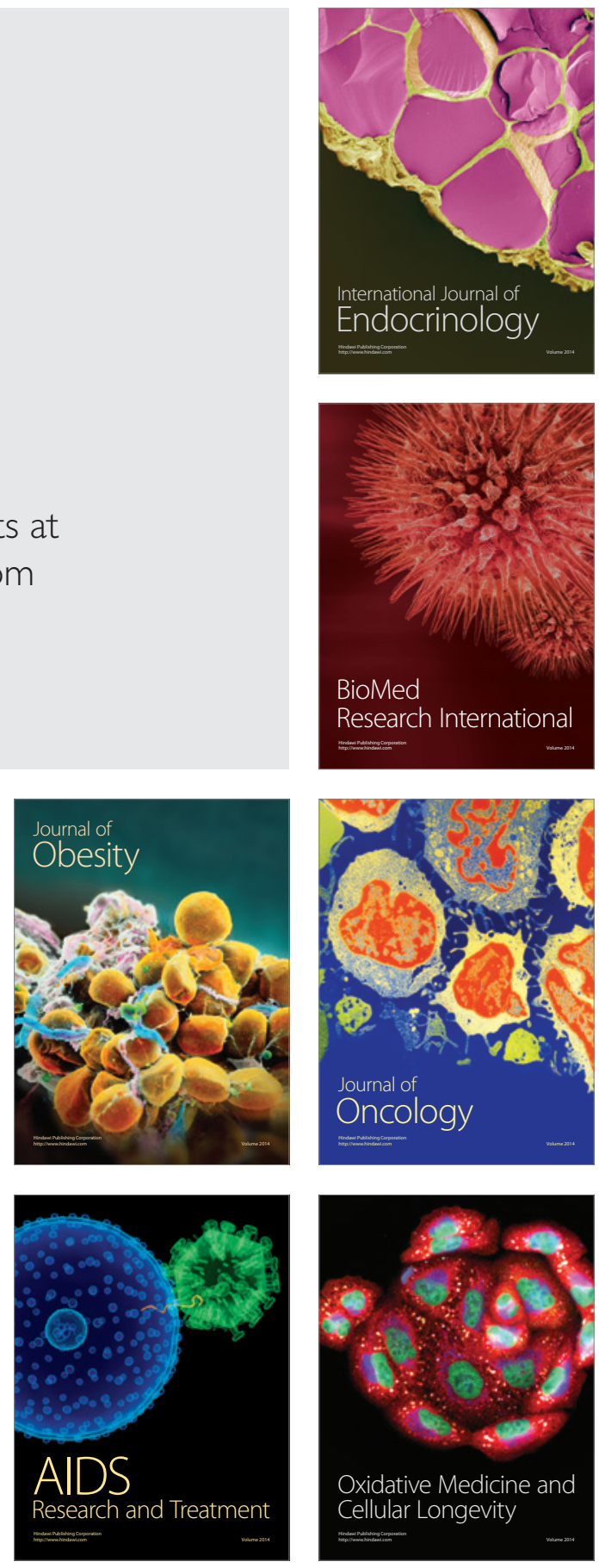